\title{
Converging Underwater and FSO Ground Communication Links
}

\author{
A. Jurado-Navas*, J.M. Garrido-Balsells, M. Castillo-Vázquez, A. \\ García-Zambrana, and A. Puerta-Notario \\ Department of Communications Engineering, University of Málaga, Campus de Teatinos, \\ 29071 Málaga, Spain \\ *navas@ic.uma.es
}

\begin{abstract}
We propose a new combined underwater-atmospheric optical communication link for providing a high speed optical connectivity between onshore and sumerge systems. New average BER expressions are derived when assuming amplify-and-forward (AF) relay.
\end{abstract}

OCIS codes: (010.1330) Atmospheric turbulence; (200.2605) Free-space optical communication 